\title{
Post-postbiological evolution?
}

\author{
Milan M. Ćirković ${ }^{1}$ \\ Astronomical Observatory of Belgrade, Volgina 7, 11000 Belgrade, Serbia
}

\begin{abstract}
It has already become a commonplace to discuss postbiological evolution in various contexts of futures studies, bioethics, cognitive sciences, philosophical anthropology, or even economics and SETI studies. The assumption is that technological/cultural evolution will soon entirely substitute for the biological processes which underlie human existence - and, by analogy, the existence of other independently evolved intelligent beings, if any. Various modes of postbiological evolution of humans have been envisioned in both fictional and discursive contexts (uploading, cyborgization, technological singularity, etc.). Little thought has been devoted so far to the question whether these postbiological modes are truly final in both logical and conceptual terms. What lies beyond the postbiological realm? Clearly, only a few radical speculations on the topic can be offered. In this paper, it is argued that in a sufficiently broad subset of scenarios for postbiological evolution there will be a kind of reverse trend: the one of reintegration with the (astro)biological universe, by that point understood in a much wider and more inclusive sense. The argument for such reintegration could be understood through the metaphor of expanding Klein bottle as a symbolic image of the post-postbiological evolutionary trajectories. This kind of trajectory will lead to the state in which products of culture are indistinguishable from the natural environment, suggesting practical consequences for our SETI efforts.
\end{abstract}

Keywords: Postbiological evolution; artificial intelligence; distributed computing; futures studies; transhumanism; philosophical naturalism; SETI

\section{Introduction}

The idea of postbiological evolution has recently emerged as mainstream - or perhaps the mainstream - thinking about the future of humanity (e.g., Kurzweil, 1999, 2005; Moravec, 1998; Dick, 2000; Pirages, 2000; Hughes, 2004; Bostrom, 2009), as well as the evolution of hypothetical extraterrestrial civilizations (Harrison, 2000; Dick, 2003; Parkinson, 2005; Ćirković \& Bradbury, 2006; Ćirković, 2012; Smart, 2009, 2012; Vidal, 2014). It is quite a reasonable and expected development, taking into account how dramatically cultural - and in particular technological - evolution has impacted not only the environment, but the physical make-up and psyche of homo sapiens itself. Since the dawn of civilization and the emergence of activities such as agriculture, medicine and civic engineering, humanity has been modifying both its physical environment and itself in an endless series of complex feedback loops. In recent years, the accelerating nature of this innovation + modification process has been recognized by many transhumanist thinkers and potentials of the near-future transition into an entirely different evolutionary regime became the focus of research in many quarters.

The term "postbiological" is nowadays used most often to refer to the general application of the convergence of nanotechnology, biotechnology, information technology and cognitive science (NBIC) to improve human performance. While this might be an oversimplification - and there are certainly technological "wild cards" in play that we are currently unaware of - it is sufficient for the needs of any crude, exploratory study such as the present one. So, while specifics may vary, we may expect that the NBIC convergence

\footnotetext{
E-mail address: mcirkovic@aob.rs.

${ }^{1}$ Also at Future of Humanity Institute, Faculty of Philosophy, University of Oxford, Suite 8, Littlegate House, 16/17 St Ebbe's Street, Oxford, OX1 1PT, UK.
} 
well describes human/terrestrial approach to the postbiological era (e.g., Canton, 2004; Bainbridge \& Roco, 2006). One important note must be made here: the locution 'evolution' too often connotes blind Darwinian processes. While it may certainly be true that classic Darwinian selectional processes will continue to be in play among cybernetic humans or within a digital substrate, there are many reasons to expect the mechanisms of change to be dramatically different. If anything, postbiological evolution could be expected to be Lamarckian in a broader sense, where intelligent agents consciously and deliberately choose their own evolutionary course.

But is postbiological evolution as it is currently conceived truly an end, the final evolutionary phase? The question is not just semantic, although it obviously has a semantic component. If postbiological is everything and all that comes after the development of transformative technologies of modernity, then by definition, there can be no post-postbiological. That definition is useless, however, in practical terms, and it could only lead to further confusions. Instead, we should regard the postbiological domain as clearly bound by some of its emerging properties: transferring previously biological processes like cognition or procreation into the machine domain and exercising complete intentional control over evolution. While human beings have exercised some degree of control over evolution for millennia (since the first development of agriculture and medicine 8,000-10,000 years ago), there are still many vagaries and genetic lotteries in our phenotype. It is to be expected that judicious use of biotechnology currently in development will soon result in complete (at least in principle) control over all expressed characters in any individual phenotype. Consequently, the entire evolution of our species will become an intentional and controlled process.

Other aspects of the NBIC convergence will concurrently step in. Notable examples discussed widely in the literature are radical life extension, mind uploading, cyborgization, cognitive and moral bioenhancement, modification for hostile environments, etc. These long-ranging options join together multiple strands of development in the process of substituting natural processes for artificial, designed and intentional alternatives. Postbiological evolution, but also other items of interest for us, was prefigured by Sir Fred Hoyle in a beautiful passage ${ }^{2}$ :

[W] ourselves are computers produced by the universe, by the long process of biological evolution, whereas the computers in our laboratory have been produced through us as an intermediary-but still by the universe. Objectionable as this conclusion will appear to many, I see little point in resisting it; it happens to be true! In growing up a child receives many rude shocks in its encounters with the world. Similarly, the human species as a whole must expect many shocks as it grows up. One of these I am convinced is that the phenomena of consciousness, of intelligence, independence, aesthetics, are going to come in ways that may seem strange to us. We must be prepared to find in the larger universe outside the Earth not only creatures very much like ourselves but wildly different ways of doing things, even "inorganic" collections of matter endowed with the sense of "justice", for example.

The same emancipation from anthropocentrism Hoyle supports here should make the concept of attractors in the parameter space of advanced technological civilizations easier to accept. If something morphologically similar to a stony crag could be found to indeed possesses a sense of justice, we could argue that obviously contingent and opportunistic morphological evolution does support cultural attractors entailing concepts like "justice“.

However, if we zoom out thing still further, we can see a catch. In the longer run, the dichotomy between biological and postbiological might become immaterial, even false - at least on the phenomenal level. This has been rather clearly expressed by a contemporary SF writer ${ }^{3}$ :

The difference between biological, biochemical, electronic, or neuro-electronic information systems, at that level of civilization-no difference, is it? Once you can rebuild yourself from the molecular level up, and out of any substance you fancy, soft or hard, stored as a pattern in a mainframe or spun out into any form of matter need calls for-no such thing as machines you can properly call by that name. It's all alive. Or all dead.

The emphasis is correctly put on the 'level of civilization' parameter. While there might be other prerequisites for such merging of biological and technological organization, it does seem to primarily depend on the overall civilization level - as proxied, for instance by Kardashev's Type, or perhaps position on the Barrow scale (which attempts to quantify control over matter in the microscopic domain). ${ }^{4}$ Last sentences of Wright's passage suggest an operational viewpoint: there is no point in trying to discern biological and postbiological, as much as there is no point in trying to discriminate between natural and artificial after some - very high - threshold civilizational level. ${ }^{5}$ On the phenomenal level, it is simply one and the same. The present paper attempts to elaborate on this particular perspective. ${ }^{6}$

This can also be put in the context of Big History: recent attempts at giving an overview of history from the Big Bang to the present. Big History examines long time frames using a multidisciplinary approach based on combining numerous disciplines and methods from science and the humanities (Nazaretyan, 2005; Stewart, 2010, 2012; Last, 2017). While Big History has observed increase of local complexity in the course of cosmological history, it has also observed the shift in the material substrate within which this complexity is manifested. This shift in substrate is rather obvious when early cosmological history is scrutinized, but there is no

\footnotetext{
${ }^{2}$ Hoyle [1964] (2005), pp. 32-33.

${ }^{3}$ Wright (2011), p. 228.

4 Barrow (1999).

5 One could speculate further that the dichotomy between form and function will disappear as well at the sufficiently high level of complexity. For an elegant fictional discussion of this issue see Robson (2005).

${ }^{6}$ Obviously, this should not be associated with any „postmodern“ nonsense about nature being at all times „socially constructed“ of the likes of those correctly identified and refuted by Koertge (1998).
} 
reason to expect the change to stop at the known biological substrate of proteins and nucleic acids. Therefore, the true Big History perspective by its very nature needs to account for postbiological evolution - that much seems clear, albeit we can hardly imagine the size of postbiological design space to be explored in such a substrate. If there is anything beyond the postbiological phase, it would be a legitimate topic for the Big History as well. Before we consider some plausible signposts on this journey, we need to delineate briefly what is not a legitimate part of this discussion.

\section{Speculative fringe possibilities}

Among the most radical and speculative possibilities considered in the pop-cultural discourse, a random and non-representative sample could read something like the following ${ }^{7}$ :

- Transference to "higher dimension", "different brane" or "other manifold".

- Entities made of "pure energy" or some variation on that.

- Field vortices in the "unified quantum field".

- Mystical transcendence to a "different mode of being" or something similar.

- Migration to another epoch via some sort of time travel.

Two points should be made here. (i) While most of these are not impossible in the sense of violating laws of physics as we know them today, some lie beyond the scope of rational analysis (the Childhood's End-type transcendence), and the other are at best extremely-low-probability type events, which cannot be taken seriously in the context of specific human future, and certainly tell us nothing about the generic evolutionary pathways of intelligent communities. While debates about whether it is possible to speak about transcendence in physical terms abound for decades if not centuries (e.g., Eddington, 1929; Nozick, 1981; Hoyle, 1983; Pollard, 1984; Davies, 1984; Rosen, 1986; Tipler, 1994; see also Young, 2012 for some older, less known examples), the content is overall rather disappointing. No operational way of making progress in these issues or a research programme in the Lakatosian sense has emerged so far. Hardly anything except subjective musings and vague speculations on the subject has ever been produced.

(ii) In each of these cases, the underlying mechanism is too vague and imprecise to allow for any sort of rational scientific analysis. The role played by assumptions such as unpredictability, contingency, and similar "veils" is crucial for these narratives. This is not to mean that some of them could not turn out to be real. Similar to the rest of science, it is not about what can be „real“; it is about what can be empirically tested and assessed within the body of our knowledge. So, while further work might enable better insight into these extremely speculative possibilities, we may still disregard them at present.

\section{Indistinguishability and the expanding Klein bottle}

The indistinguishability thesis: sufficiently advanced technology is indistinguishable from its natural, in particular astrophysical/astrobiological, environment. It follows that, from an external perspective - for example from the point of view of our feeble SETI attempts to detect advanced civilizations in the Milky Way - it is very difficult if not impossible to be certain about intentional, artificial origin of any particular signal. Thus, if true, the indistinguishability thesis has a practical significance for the way we search for advanced civilizations. Clearly, this implies a sort of re-integration of advanced technology with the set of algorithms already created by nature. An external observer would be hard pressed to find any discriminative feature between non-intentional ('natural') and intentional ("artificial") features of the observed spacetime region. The difference between conventionally "natural" and conventionally "artificial" has almost monotonously increased over the course of human history so far. At some point, these will begin to re-converge.

It is much more uncertain to speculate what would be seen by an internal observer. Conventional properties of individual observer have already been undermined in the early stages of postbiological evolution we see around us; therefore, it is difficult to extend its perspective into any hypothetical subsequent phase. While its phenomenology and manifestations could appear as either biological or non-biological (e.g., machines), for most of the time and most of the values of the parameter space, it will encompass both. Many models are possible which could represent this indistinguishability. One might envision biocomputing so energy-efficient and widely distributed that it is literally woven into the biosphere of a planet including grass, marine flora, coral reefs, etc. Alternatively, we could have a "technosphere" built entirely from "smart dust", the concept described in a premier research journal in the field of textile technology (Farrer, 2010), and ascribed to a 1964 novel by Stanislaw Lem, in which it is a product of natural selection on a distant planet. ${ }^{8}$ Many of stable civilization configurations are likely to be singletons (Bostrom, 2006), supposedly offering solutions to the long-term coordination problems.

The metaphor of the Klein bottle (Fig. 1) presents a way of thinking about separation and re-joining of the artificial and the natural. It is a smooth, non-orientable, 2-D surface, curved into itself in such manner that there is no distinction between an inner and outer volume (or inner or outer surface). This favourite topological toy represents 3-D generalization of the even more popular Moebius strip. If we consider the width of the Klein bottle as a metaphor for the scope or diversity of (biological + cultural) evolution, we could conceive each "cycle“ as a process in which a smaller subsection of this scope generates the rest, including itself.

\footnotetext{
${ }^{7}$ For a small sample of the huge SF literature on the topic - some of which will be considered in slightly more detail below - see Clarke (1953, 1956, 1968); Lovecraft [1936] 1999; Schroeder (2001, 2005); Reynolds (2003, 2005). Some critical accounts can be found in Nicholls (2000).

8 The novel is The Invincible (Lem [1964] 1973).
} 


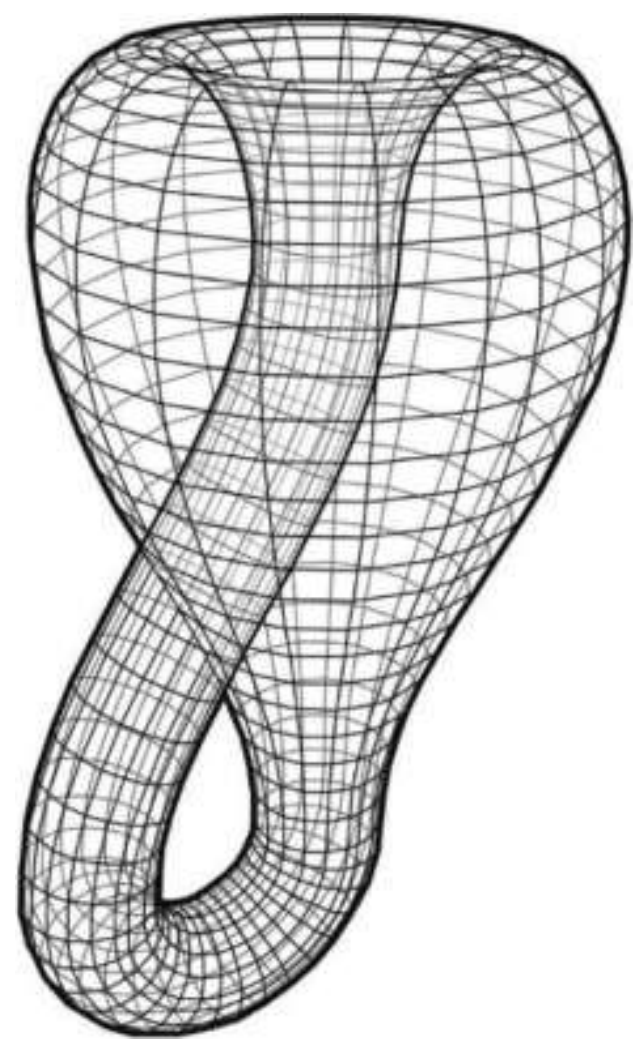

Fig. 1. Klein bottle, the most famous example of a non-orientable surface with no boundary. An evolutionary trajectory could map smaller part of the flow to the main cross-section while switching from internal to the external side and back - as a metaphor for rejoining postbiological evolution with nature "turned inside out".

There are no sharp boundaries, and we could imagine that the wide portion of the bottle is much wider, even orders of magnitude, than the narrow „bottleneck“. In the wider parts dominant processes are - to an external observer - allegedly „natural“ or biological and in the narrower parts they are technological or post-biological; only apprehension of the whole allows for the key insight that they are not really separable, but completely entangled. If we further imagine many cycles of motion over bottle surface and use the winding number as a measure of time, we shall perceive this entanglement in both terms of space (evolutionary diversity) and time. This does not mean that any postbiological trajectory leads to such a re-convergence in the post-postbiological domain; after all, the Klein bottle is manifestly not axially symmetric.

Of course, a post-postbiological stage of the universal evolution should not be regarded as some simplistic "return to Mother Nature" (or any number of similar ideological slogans). In a sense, it represents the real „end of nature“, since it entails the loss of the specific ontological status embedded in the identifier „natural“. ${ }^{9}$ Of course, it does not mean the loss of the phenomenal spectrum we commonly associate with non-intentional evolutionary process - if anything, post-postbiological stage will present regaining some parts of that spectrum which could be lost in the interim postbiological phase. Due to the postbiological emphasis on information, we could expect that these parts were retained in the sense of potentiality, similar to the genome of extinct species, preserved for subsequent de-extinction. We shall see some of the literary examples below in some detail, but many more are available. In the fictional universe of Ridley Scott's celebrated movie Blade Runner, owls are extinct (together with most of other animals species), and so the owl-like entity in possession of a corporate boss is an artificial entity, essentially a robot, no matter how eerily "real" it looks. This is a postbiological solution; a step further, post-postbiological solution would be to create an ecosystem in which owls could be re-evolved again, or perhaps made de-extinct in accordance with their preserved or reconstructed genomic information. Such solution could look like an extravagant luxury or wasting huge resources - but the only reason for such a conclusion is that we tend to perceive only a single function for owls in the fictional universe (to serve as glorified pets). There are many contexts in which postpostbiological solution is much more efficient in terms of time, resources, and both intended and unintended benefits.

\section{Motivation and drives}

What could be the motivation and major drives leading to the postbiological - post-postbiological transition? While they are certainly extremely difficult to gauge in advances, some of the possibilities include:

\footnotetext{
${ }^{9}$ In contrast to some of the contemporary alarmist narratives, e.g., McKibben (1989).
} 
- The challenge of heterogeneous habitats of extrasolar planets and those encountered in the course of space expansion in general. Since the natural (= non-intentional) evolution has been at work there for billions of years, all these widely heterogeneous sets of algorithms should be co-opted for intelligent species' use.

- (In)efficiency of integrating the remnants of the previous, biological phase of evolution into purely non-biological substratum. In contrast, using nature itself for computational purposes (as in various forms of widely distributed computing, cf. Hutchins, 1995) might turn out to be the most advantageous route.

- The expansion of the universe: while it can be shown that a cosmologically large volume could be reached by advanced technological civilizations (Armstrong \& Sandberg, 2013; Olson, 2015), it is not obvious how quickly these huge volumes of space can be "technologized". Clearly, it would make much sense to use the already available, independently evolved structures and even "recipes" created by natural evolution (under the assumption that local resources are not already marshalled and controlled by local technological civilizations). Such co-opting makes more sense in the case of limited timescales than constructing the entire civilizational infrastructure ab initio.

- The increase in control over matter below the nanometre level; in particular, efficient femtotechnology (manipulation of nucleons within an atomic nucleus) leading to cheap transmutation of elements. This could lead to a situation in which it is cheaper to substitute only individual, precisely determined components of complex natural systems - e.g., living beings - to obtain a desired functional objective than to build the whole from scratch.

- Ethical reasons, like reaching a higher moral valuation of the natural and the evolved, or putting stronger emphasis on the ontological diversity within a civilization. Since it is difficult to predict anything about ethics of advanced cultures, there is great latitude in formulating possible reasons of this kind.

- Especially interesting type of ethical motivation could be the desire to achieve as perfect autonomy for individual actors within the civilization as possible. This would include all types of primitivist, nostalgic, recidivist, culturally-anarchist, pan-cosmist, etc., factions and schools of thought, as well as groups and regions within the civilization which for one reason or another (being too distant or being kept as „wilderness preserves“, parts of long-term scientific experiments, etc.) have not been integrated into the postbiological mainstream. I shall discuss a fiction elaboration of this motivation by Karl Schroeder in the next section.

- If it turns out that interstellar panspermia is ubiquitous in the Galaxy, this would arguably mean that the total set of evolutionary algorithms is much larger and more flexible than hitherto supposed, justifying their "off the shelf" usage in reintegrated naturaltechnological systems.

Each item requires a separate analysis, which is far beyond the scope of the present paper. For the moment, it is enough to establish that relevant drives do exist and, at the very least, may play a role in particular scenarios of postbiological evolution. As our insight into particulars of postbiological evolution increases, we shall be able to streamline and specify and prune these different motivations we speculate now about.

\section{Reintegration with biological: two fictional examples}

As it often happens, this possibility has been prefigured in the artistic discourse, particularly in the domain of science fiction, sufficiently generalized. I shall analyse here two pertinent examples, Stanislaw Lem's story-essay "New Cosmogony" (from the 1971 experimental anthology A Perfect Vacuum) and the novel Lady of Mazes by contemporary Canadian author Karl Schroeder (Lem [1971] 1999; Schroeder, 2005). While the two authors could hardly be more dissimilar, I shall demonstrate how the general theme of post-postbiology plays out in a surprisingly similar structural manner in their fictional universes. ${ }^{10}$

In a brilliant, but poorly known "mock essay" of Stanislaw Lem, entitled "The New Cosmogony" in his idiosyncratic anthology $A$ Perfect Vacuum, we can find a serious attempt - the first after Stapledon's (1937) Star Maker - at outlining the shape of truly advanced intelligent communities. ${ }^{11}$ Lem, perhaps unjustly known more as a writer than as an astrobiologist and a fascinating philosopher of science, has time and again shown his boldness to tackle the issues related to life and mind in the universe. In contrast to the timid observer of Stapledon's novel, Lem's narrator is a modern-day Nobel-prize winner, Prof. Alfred Testa, ${ }^{12}$ a somewhat cynical physicistphilosopher who describes his "trivial" solution of Fermi's Paradox. Why don't we perceive artefacts and engineering of supercivilizations billions of years older than ours? No, no, no, he says, we do! - the laws of physics themselves are product of their engineering, of the Great Game, played on largest possible spatial and temporal scales. The Players' pool of knowledge is so vast and their capacities so overwhelming, that only "by their fruits ye shall know them" - and those are the (effective) laws of nature ${ }^{13}$ :

If one considers "artificial" to be that which is shaped by an active Intelligence, then the entire Universe that surrounds us is

\footnotetext{
${ }^{10}$ On the individual level, the issue of (re)integration with the biological has much longer SF ancestry, which could be traced even to Mary Shelley's original Frankenstein (Shelley [1818] 1994). In the discourse of modern SF, Isaac Asimov's famous novelette The Bicentennial Man, could be understood as the personal metaphor of gradual erasing the boundaries between what is robotic - hence in a sense postbiological - and what is human (Asimov [1976] 1992). Finally, in Alastair Reynolds' „ Poseidon's Children“ trilogy, for the most part „invisible“ character of Eunice Akinya has started as a rather crude piece of software, expert-system-level; after many rounds of improvement by both human and extraterrestrial agencies, as well as self-improvement is revealed at the end as the perfect blend of biological and AI worlds, necessary for the wider dramatic rendering of the cosmic fate of humanity (see in particular Reynolds, 2015).

${ }^{11}$ Lem [1971] (1999).

12 The name is, perhaps, an allusion to Paul Valéry's Mr. Edmond Teste, a perfect intellectual (Valéry 1989). Monsieur Teste's motto is: "Que peut un homme?," meaning "Of what is man capable?" If we substitute "an intelligent being" for "man", we obtain the keystone question of "The New Cosmogony".

${ }^{13}$ Valéry (1989), p. 208, emphasis in the original.
} 
already artificial... Instrumental technologies are required only by a civilization still in embryonic stage, like Earth's. A billionyear-old civilization employs none. Its tools are what we call the Laws of Nature. Physics itself is the "machine" of such civilizations!

He continues to explain the hierarchical levels of various "physicses" and the strategies of reconstructing, using game-theory methods, the evolutionary pathway of our own effective laws. The very difference between the natural and the artificial is thus erased at some point in the course of cultural evolution. The answer to Fermi's question Where are they? is everywhere, literally. Rejecting scientific realism need not necessarily mean the defeat of science - it could (in an admittedly contrived context) be the ultimate vindication of its power. To think that "The New Cosmogony" predates - by decades! - such relevant scientific ideas and concepts as distributed computing, cosmological computational bounds, the Internet-of-things, the holographic principle, not to mention multiple vacuums of superstring theory, evolving values of the fundamental constants of physics or eternal inflation, is truly flabbergasting. ${ }^{14}$

What if the local control over the environment is complete, but there are no capacities or motivation or time to extend it into the cosmological domain? Lady of Mazes is a spectacular, colourful journey through the posthuman world of the far future, an archipelago containing alien species, superintelligent AIs, astroengineering projects and an extraordinary level of sentient control over environment. It does not mean that there are no problems and Schroeder's world is anything but utopian - the problems and conflicts are elevated to quite another level, however, even intuitively difficult to comprehend by observers like us, the present-day humans.

The key element of Schroeder's plot is the fictional metatechnology of "tech locks", which enables any user to strictly define the level of technological advancement in a given surroundings. Without going into any specifics, the tech locks enable some users to live in a hunter-gatherer society, others to experience slave-holding empires, or superurban sprawls, or global meditation gardens, or cyberpunk intrigues - it is all possible, depending on the tech lock settings. The idea that particular technologies are the main factor limiting stability and durability of particular cultural and social systems which Schroeder here promotes is in itself extremely interesting and deserving of deeper scrutiny. What is important from the point of view of the present discussion, however, is that the tech locks are "nature locks“ as well, since they delimit the role of what is phenomenally (although not necessarily ontologically) natural. ${ }^{15}$ Therefore, Raven - one of the major protagonists - is capable of exercising his radical autonomy by living in traditional „harmony with nature' similar to his (real or imagined) Native American forefathers, while some of the other protagonists share more modern cultural systems without imperiling each other's values in any meaningful sense. The freedom of individual actors reaches unprecedented level due to reintegration of the "natural' and the 'artificial'.

What is really „unnatural“ in Schroeder's world is the intrusion of an external force, which turns out to be a powerful AI, wishing essentially to "roll back" the wheel of history (as far as we - or the protagonists of the novel - are able to discern its motivation at all) to a totalitarian "ordered' state with a common, mandatory form of reality. There is a whole fascinating layer of the narrative in Lady of Mazes dealing with this societal and political aspect of the conflict between the conventional postbiological and post-postbiological views which I cannot investigate here in detail.

The relevance to the present topic is obvious: at least when discussing advanced cultures, human, posthuman, or extraterrestrial, the only meaningful perspective is the phenomenal one. We are engaging in SETI searches exactly because we hope to detect anomalous phenomena which are in this sense (and in the vernacular as well) 'unnatural'. A signal containing prime numbers, a rectangular object transiting its parent star, a Dyson sphere, a perfect black monolith of 1:4:9 proportions, and myriad similar concepts are examples of potentially observable phenomena deemed impossible or extremely unlikely to arise by non-intentional, natural processes. While we might be mistaken in some particular cases, ${ }^{16}$ and the details of our predictions might be wrong, the conjunction of various lines of argument for intentionality offers as good a guideline for scientific evidence as anything else.

In accordance with the central „Copernican“ duality, what is relevant for our SETI efforts is eo ipso relevant for assessing the future of humanity. If we conclude that our SETI projects have been unsuccessful so far because all extraterrestrial civilizations self-destruct very soon after inventing nuclear weapons, the posterior probability of our own self-destruction in a nuclear holocaust dramatically increases. In the particular case of postbiological evolution and its hypothetical extension(s), the more we deem some particular bunch of trajectories to be cultural attractors, the more reasons we have to expect the future of humanity to follow a trajectory in that bunch. Therefore, if we find theoretical or observational evidence for widely distributed computing or any similar post-postbiological trajectory outlined above, we can meaningfully increase the probability of some future trajectories for humanity and decrease the probability of others. This is yet again (cf. Dick, 2003) a path along which our SETI efforts have very practical significance for thinking about the future of humanity.

\section{Discussion and prospects}

We consider the possible next phase in evolutionary trajectories of advanced technological civilizations, on Earth and beyond. While it is obviously impossible to offer any precise view of the trajectory of postbiological evolution, it is not premature to boldly

\footnotetext{
${ }^{14}$ Compare, for instance, Carroll (2006). While the idea of fundamental "constants" changing with cosmic time was around since Dirac's "large number hypothesis" in 1930s, the real impetus to study such theories came from both observational and theoretical work in 1990s; see Uzan (2003) and references therein. For the closest recent ideas about life and intelligence on truly cosmological scale, see Smart (2009).

${ }^{15}$ Ursula Pflug touches upon this key point in her thought-provoking review of The Lady of Mazes for Strange Horizons: „In Raven the coyote would have wished me good morning, in English, instead of in its own way, which was to stop on its trail and look at me and my infant son awhile before it ambled on. In Raven's worlds, no one would even think to ask the entrancing question: is it the coyote's field or mine?" (http://strangehorizons.com/non-fiction/reviews/lady-of-mazes-by-karlschroeder/, last accessed June 14, 2017).

${ }^{16}$ For an amusing example, see the plot of Bioy Casares [1940] (1996).
} 
speculate about its possibilities and long-term outcomes. At the very least, this could frame the discussion and prepare ground for future more informed and detailed accounts, based presumably on both new theoretical insights and increased capacity for numerical simulations of cultural evolution. The design space of the postbiological evolution is certainly huge even in comparison to the morphological space of the underlying biological substratum; still, it is to be expected that this design space is characterized by at least some non-ergodicity and cultural attractors. A separate, though extremely intriguing, question is whether the relative size of viable (or long-term-survivable) part of the entire postbiological design space is proportionally large or not; it is possible that constraints such as dysgenic pressures and existential risks posed by superintelligent AI or some entirely unconceived future technologies play a much stronger role in limiting the viable chunk of the design space.

As argued above, there are reasons to believe that reintegration with the biological nature, understood in the widest and most liberal manner, is one such attractor in the postbiological design space. The indistinguishability thesis may give us a handle to sharpen our intuitions on the subject and formulate more precise and ultimately testable hypotheses. Such reintegration would provide a model for many intermediate- and low-scale processes occurring in various parts of civilization (generally understood, not necessarily in spatial terms, but also in terms of whatever parameter space is relevant for such advanced forms of organization). At first, ignoring metaphysical debates about what is really natural, we may reasonably proceed to consider such reintegration as a new transition, into the post-postbiological domain. In the longer run, we might be able to proceed to actually make these traditionally metaphysical questions an object of practical concern and even decision- and policy-making.

While many qualms and criticisms could be expected about the outline sketched here, the basic point is hopefully widely acceptable: we do not have any reason to believe that the postbiological domain, as currently debated, is the true endpoint of evolution. On the contrary: barring extinction, it is to be expected that the series of challenges posed by both the cosmic environment and social structure of any civilization will, in the fulness of time, prompt any number of structural responses. It is to be hoped that multidisciplinary research at the confluence of futures studies, AI, astrobiology, and social science will provide further means to assess such speculative ideas about the future evolution, on Earth and beyond.

\section{Acknowledgements}

Useful comments of an anonymous referee are hereby acknowledged. The author wishes to thank George Dvorsky, John Smart, Karl Schroeder, Aleksandar Obradović, Milan Stojanović, and Srdja Janković who offered helpful suggestions which immensely improved a previous version of this manuscript. Dušan Pavlović has kindly helped in obtaining some of the literature. The author acknowledges financial support from the Ministry of Education, Science and Technological Development of the Republic of Serbia through the projects \#ON176021 and \#ON179048.

\section{References}

Armstrong, S., \& Sandberg, A. (2013). 'Eternity in six hours: Intergalactic spreading of intelligent life and sharpening the Fermi paradox. Acta Astronautica, 89, 1-13.

Asimov, I. [1976], 1992. The Bicentennial Man, in The Complete Stories, Volume 2 (Doubleday, New York), pp. 568-604.

Bainbridge, W. S., \& Roco, M. C. (2006). Managing nano-bio-info-cogno innovations. Dordrecht: Springer.

Barrow, J. D. (1999). Impossibility: The limits of science and the science of limits. Oxford: Oxford University Press.

Bioy Casares, A., [1940] 1996. La invención de Morel (Penguin Books, London).

Bostrom, N. (2006). What is a singleton? Linguistic and Philosophical Investigations, 5, 48-54.

Bostrom, N. (2009). In J.-K. Berg Olsen, E. Selinger, \& S. Riis (Eds.). The future of humanity, in new waves in philosophy of technology (pp. 186-216). New York: Palgrave McMillan.

Ćirković, M. M., \& Bradbury, R. J. (2006). Galactic gradients, postbiological evolution and the apparent failure of SETI. New Astrophysics, 11, 628-639.

Ćirković, M. M. (2012). The astrobiological landscape: Philosophical foundations of the study of cosmic life. Cambridge: Cambridge University Press.

Canton, J. (2004). Designing the future: NBIC technologies and human performance enhancement. Annals of the New York Academy of Sciences, 1013, 186-198.

Carroll, S. M. (2006). Is our universe natural? Nature, 440, 1132-1136.

Clarke, A. C. (1953). Childhood's end. New York: Ballantine Books. Clarke, A. C. (1956). The city and the stars. London: Frederick Muller Ltd. Clarke, A.C., 1968, 2001. A Space Odyssey (Hutchinson, London).

Davies, P. C. W. (1984). God and the new physics. New York: Simon \& Schuster. Dick, S. J. (2000). Interstellar humanity. Futures, 32, 555-567.

Dick, S. J. (2003). Cultural evolution, the postbiological universe and SETI. International Journal of Astrobiology, 2, 65-74.

Eddington, A. S. (1929). Science and the unseen world. Macmillan: New York.

Farrer, J. (2010). Smart dust: Sci-Fi applications enabled by synthetic fiber and textiles technology. Textile, 8, $342-347$.

Harrison, A. A. (2000). Slow track, fast track, and the 'Galactic Club'. Futures, 32, 569-579. 
Hoyle, F. (1983). The intelligent universe. London: Michael Joseph Limited.

Hoyle, F., [1964] 2005. Of Men and Galaxies (Prometheus Books, Amherst).

Hughes, J. (2004). Citizen cyborg: Why democratic societies must respond to the redesigned human of the future. New York: Basic Books.

Hutchins, E. (1995). Cognition in the wild. Cambridge: The MIT Press.

Koertge, N. (1998, ed.) A house built on sand: Exposing postmodernist myths about science. In Oxford: Oxford University Press.

Kurzweil, R. (1999). The age of spiritual machines: When computers exceed human intelligence. New York: Viking.

Kurzweil, R. (2005). The singularity is near: When humans transcend biology. New York: Viking.

Last, C. (2017). Big historical foundations for deep future speculations: Cosmic evolution, atechnogenesis, and technocultural civilization. Foundations of Science, 22, 39-124.

Lem, S., [1964] 1973. The Invincible (Sidgwick and Jackson, London).

Lem, S., [1971] 1999. The New Cosmogony, in A Perfect Vacuum (trans. by M. Kandel, Northwestern University Press, Evanston), 197-227.

Lovecraft, H.P., [1936] 1999. The Shadow Out of Time, in The H. P. Lovecraft Omnibus I: At the Mountains of Madness (Harper Collins, London).

McKibben, B. (1989). The end of nature (Anchor, New York).

Moravec, H. (1998). Robot: Mere machine to transcendent mind. Oxford: Oxford University Press.

Nazaretyan, A. P. (2005). Big (Universal) history paradigm: Versions and approaches. Social Evolution History, 4, 61-86.

Nicholls, P. (2000). Big dumb objects and cosmic enigmas: The love affair between space fiction and the transcendental. In G. Westfahl (Ed.). Space and Beyond. The Frontier Theme in Science Fiction (pp. 11-23). Westport: Praeger.

Nozick, R. (1981). Philosophical explanations. Cambridge: Harvard University Press.

Olson, S. J. (2015). Homogeneous cosmology with aggressively expanding civilizations. Classical and Quantum Gravity, 32 , 215025.

Parkinson, B. (2005). 'The carbon or silicon colonization of the universe? Journal of the British Interplanetary Society, 58, 111116.

Pirages, D. (2000). Diversity and social progress in the next millennium: An evolutionary perspective. Futures, 32, 513-523.

Pollard, W. G. (1984). Rumors of transcendence in physics. American Journal of Physics, 52, 877-881.

Reynolds, A. (2003). Absolution gap. London: Gollancz.

Reynolds, A. (2005). Pushing ice. London: Gollancz.

Reynolds, A. (2015). Poseidon's wake. London: Gollancz.

Robson, J. (2005). Natural history. New York: Bantam Books.

Rosen, J. (1986). No rumors of transcendence in physics. American Journal of Physics, 54, 700-702.

Schroeder, K. (2001). Ventus. New York: Tor Books.

Schroeder, K. (2005). Lady of Mazes. New York: Tor Books.

Shelley, M. [1818], 1994. Frankenstein (Dover Publications, Mineola, New York).

Smart, J. M. (2009). Evo devo universe? a framework for speculations on cosmic culture. In S. J. Dick, \& M. L. Lupisella (Eds.). Cosmos and culture: Cultural evolution in a cosmic context (pp. 201-295). Washington: NASA.

Smart, J. M. (2012). The transcension hypothesis: Sufficiently advanced civilizations invariably leave our universe, and implications for METI and SETI. Acta Astronautica, 78, 55-68.

Stapledon, O. (1937). Star maker. London: Methuen.

Stewart, J. E. (2010). The meaning of life in a developing universe. Foundations of Science, 15, 395-409.

Stewart, J. E. (2012). The future of life and what it means for humanity. Foundations of Science, 17, 47-50.

Tipler, F. J. (1994). The physics of immortality. New York: Doubleday.

Uzan, J.-P. (2003). The fundamental constants and their variation: Observational and theoretical status. Reviews of Modern Physics, 75, 403-455.

Valéry, P. (1989). Monsieur teste (translated by J. Mathews). Princeton: Princeton University Press.

Vidal, C. (2014). The beginning and the end: The meaning of life in a cosmological perspective. New York: Springer.

Wright, J. C. (2011). Count to a trillion. New York: Tor Books.

Young, G. M. (2012). The russian cosmists: The esoteric futurism of nikolai fedorov and his followers. Oxford: Oxford University Press. 